\title{
On the particular importance of vibrational contributions to static electric properties of model linear molecules under spatial confinement ${ }^{\dagger}$
}

\author{
Robert Zaleśny, ${ }^{*, a, b}$ Robert W. Góra, ${ }^{a}$ Josep M. Luis ${ }^{c}$ and Wojciech Bartkowiak ${ }^{*, a}$
}

\author{
Received Xth $X X X X X X X X X X 20 X X$, Accepted Xth $X X X X X X X X X 20 X X$ \\ First published on the web Xth $X X X X X X X X X X 200 X$ \\ DOI: 10.1039/b000000x
}

The influence of the spatial confinement on the electronic and vibrational contributions to longitudinal electric-dipole properties of model linear molecules including $\mathrm{HCN}, \mathrm{HCCH}$ and $\mathrm{CO}_{2}$ is discussed. The effect of confinement is represented by twodimensional harmonic oscillator potential of cylindrical symmetry, which mimics the key features of various types of trapping environments like, for instance, nanotubes or quantum well wires. Our results indicate that in general both (electronic and vibrational) contributions to (hyper)polarizabilities diminish upon spatial confinement. However since the electronic term is particularly affected the relative importance of vibrational contributions is larger for confined species. This effect increases also with the degree of anharmonicity of vibrational motion.

Effects of high pressure and spatial confinement on electronic structure play a major role in many areas of physics and chemistry, including nanotechnology, astrophysics and physics of plasma. ${ }^{1-12}$ Response of an atomic or molecular system to a strong confining environment found in endohedral complexes, mesoporous materials and molecular containers or subjected to extreme pressures, like those induced in the diamond-anvil, can be theoretically described, to a first approximation, considering a box of penetrable or impenetrable walls. ${ }^{2-8}$ The corresponding confining potential, which leads to the orbital compression (deformation) of guest molecule or atom, can be introduced in the Hamiltonian in the form of one electron operator:

$$
H=H^{0}+V_{\text {conf }},
$$

where $H^{0}$ is the Hamiltonian of isolated molecule. Such an approach, encompassing several types of model confining potentials (penetrable spherical box, harmonic oscillator as well as Gaussian confining potential), was recently applied in a series of $a b$ initio studies concerning the influence of spatial restriction on the electric-dipole properties, which is also the subject of this communication. The set of dipolar molecules

\footnotetext{
$\dagger$ Electronic Supplementary Information (ESI) available: details of electronic and vibrational structure calculations. See DOI: 10.1039/b000000x/

${ }^{a}$ Department of Physical and Quantum Chemistry, Faculty of Chemistry, Wroctaw University of Technology, Wybrzeze Wyspiańskiego 27, 50-370 Wroctaw, Poland

${ }^{b}$ Department of Chemistry, Faculty of Natural Sciences, Matej Bel University, Tajovského 40, SK-97400 Banská Bystrica, Slovak Republic.

${ }^{c}$ Institute of Computational Chemistry and Catalysis and Department of Chemistry, University of Girona, Campus de Montilivi, 17071 Girona, Catalonia, Spain

*E-mail: robert.zalesny@pwredu.pl orwojciech.bartkowiak@pwredu.pl
}

and molecular complexes studied thus far includes $\mathrm{LiH}, \mathrm{HF}$, $\mathrm{LiF}, \mathrm{HCl}, \mathrm{HCCCN}, \mathrm{HArF}$ and linear complexes of HCN. ${ }^{13-20}$ The effect of spatial confinement on the electric properties was also analyzed for the $\mathrm{LiH}, \mathrm{HF}, \mathrm{LiF}, \mathrm{HCl}$, and $\mathrm{HCCCN}$ molecules based on the supermolecular approximation, ${ }^{18-20}$ in which case the molecular cages, represented by either carbon nanotubes or nanotube-like helium clusters, were used to render the confining environment.

In this paper we extend our previous efforts by studying the effects of confining potential on the vibrational contributions to molecular polarizability $(\alpha)$, first $(\beta)$ and second hyperpolarizability $(\gamma)$ of three polyatomic molecules: $\mathrm{CO}_{2}$, $\mathrm{HCN}$ and $\mathrm{HCCH}$. It is fair to mention that the vibrational (hyper)polarizabilities of these molecules in an unconfined environment were studied by other authors. ${ }^{21-27}$ In the case of atomic and molecular systems, the properties in question are quantitative measure of several linear and nonlinear optical processes at microscopic level. The significance of this subject arises from the fact that understanding of the electrical properties of molecular systems in confining environments, in contrast to atoms, ${ }^{2-5}$ is still very limited. In particular, the effects on the vibrational electrical properties have not been discussed in the literature, except some preliminary results obtained recently for HCCCN molecule. ${ }^{15}$ Unlike atoms, molecules possess the rotational and vibrational degrees of freedom. This leads to a more complicated picture of the linear and nonlinear electrical response of molecules (even diatomics) in the presence of external perturbations (e.g. electric fields). The importance of the vibrational contributions to the molecular (hyper)polarizabilities is nowadays well 
Tab. 1 Changes in bond distances ( $\Delta_{i-j}$ given in Angstroms) with respect to geometry optimized without the presence of confining potential $(\omega=0)$

\begin{tabular}{|c|c|c|c|c|c|}
\hline \multirow[t]{2}{*}{$\omega$} & \multirow{2}{*}{$\frac{\mathrm{CO}_{2}}{\Delta_{\mathrm{C}-\mathrm{O}}}$} & \multicolumn{2}{|c|}{$\mathrm{HCCH}$} & \multicolumn{2}{|c|}{$\mathrm{HCN}$} \\
\hline & & $\Delta_{\mathrm{C}-\mathrm{C}}$ & $\Delta_{\mathrm{C}-\mathrm{H}}$ & $\Delta_{\mathrm{C}-\mathrm{N}}$ & $\Delta_{\mathrm{C}-\mathrm{H}}$ \\
\hline 0.08 & -0.003 & -0.005 & -0.004 & -0.004 & -0.004 \\
\hline 0.16 & -0.012 & -0.019 & -0.015 & -0.015 & -0.014 \\
\hline 0.24 & -0.024 & -0.036 & -0.030 & -0.030 & -0.028 \\
\hline
\end{tabular}

recognized. ${ }^{28,29}$ It has been shown that these contributions in some instances may dominate over the electronic counterparts. The theoretical prediction of the vibrational corrections to the (hyper)polarizabilities of polyatomic molecules is still challenging for quantum chemistry methods. Usually, the Born-Oppenheimer (BO) approximation is the starting point for computations of the electric properties of molecules (see Ref. 30,31 for an example of an alternative nonadiabatic approach for computing (hyper)polarizabilities). Within BO approximation the total energy may be separated into terms related to electronic and nuclear degrees of freedom. Hence, molecular properties may also be divided into the corresponding electronic and vibrational contributions.

In this communication, in order to study the effect of spatial confinement on the vibrational contributions to static electric properties, we employed two approaches. Namely, the perturbation method of Bishop and Kirtman was used to determine vibrational corrections based on the double harmonic approximation (hereafter referred to as harmonic contributions) ${ }^{25,32,33}$ while the finite-field nuclear relaxation (FF-NR) method ${ }^{34,35}$ was employed to determine nuclear relaxation hyperpolarizabilities. In the case of the FF-NR method the vibrational correction to electrical property $\left(P^{\mathrm{VIB}}\right)$ is interpreted in terms of contributions due to the shift of the equilibrium geometry in the presence of an external electric field (the so-called nuclear relaxation contribution, $P^{\mathrm{NR}}$ ) and contribution due to the change of the shape of the potential energy surface (the curvature contribution, $P^{\text {curv }}$ ). A comparison and detailed analysis of these two schemes has been discussed many times in the literature. ${ }^{36,37}$ The $P^{\text {curv }}$ contributions are usually smaller than the $P^{\mathrm{NR}}$ contribution and are not computed here. It is worth to note that the FF-NR method yields first and second hyperpolarizability including electrical and mechanical anharmonicity. All calculations reported in this communication correspond to the static limit (time-independent electric fields are considered). The pure electronic contributions were calculated at the equilibrium geometries using the finite-field method. ${ }^{38}$ The choice of external electric field $(F)$ strength is particularly important for the numerical evaluation of energy and property derivatives using finite differences. Thus, in this communication, we use the Romberg-Rutishaser scheme ${ }^{39}$ to determine pure electronic $\left(P^{\mathrm{EL}}\right)$ and vibrational (nuclear relaxation, $P^{\mathrm{NR}}$ ) contributions to the molecular (hyper)polarizabilities, and to control the numerical stability of the results. The linear molecules investigated here $\left(\mathrm{CO}_{2}, \mathrm{HCCH}\right.$ and $\left.\mathrm{HCN}\right)$ were oriented along $z$-axis in all calculations. The FF-NR treatment was applied only for the dominant longitudinal (along $z$-axis) components of the static (hyper)polarizabilities.

The model two-dimensional harmonic potential was centered at the molecular axis: ${ }^{2,8,14-16,18}$

$$
V_{\text {conf }}\left(r_{i}\right)=\frac{1}{2} \omega^{2} r_{i}^{2}=\frac{1}{2} \omega^{2}\left(x_{i}^{2}+y_{i}^{2}\right)
$$

This type of model confining potential allows to mimic a smoothly varying strength of spatial restriction by setting the value of $\omega$, being the quadratic force constant of the applied harmonic potential. Similarly to our previous study, the range of $\omega$ values considered in this communication was adjusted to render the exchange repulsion of linear molecules encapuslated in carbon nanotube and varies from 0 to 0.24 au. ${ }^{15}$ The geometries of the $\mathrm{CO}_{2}, \mathrm{HCCH}$ and $\mathrm{HCN}$ molecules were fully optimized in vacuum as well as in the presence of the confining potential using the $\operatorname{CSSD}(\mathrm{T}) /$ aug-cc-pVTZ approach. The same method was applied for calculations of the electrical properties of the investigated molecules. The geometry relaxations in the external confining potential (and electric field) were carried out with the aid of procedure developed by Luis et al. ${ }^{35}$ In this approach the geometry optimization is performed by means of the Broyden-Fletcher-Goldfarb-Shano method based on the numerical energy gradients calculated employing the GAUSSIAN 09 package. ${ }^{40}$

Since our theoretical predictions cannot be directly compared with the available experimental data, we would like to present them in the broad context of experiences and knowledge emerging from computational and experimental studies concerning the effects of spatial restriction or high pressure on the individual molecules and molecular materials. It is obvious that vibrational contributions to (hyper)polarizabilities of molecules must be particularly influenced by the changes in molecular geometry. Indeed, it has been shown that high pressure induces significant changes of position and intensity of vibrational bands in IR and Raman spectra of molecules in various molecular materials. The direction of the pressureinduced shifts and broadening of vibrational bands depends on the balance between short-range repulsive and long-range attractive intermolecular forces. ${ }^{41-48}$ Our theoretical approach based on imposing the model confining potential is related to the situation when strong repulsion and consequent electron density deformation is the dominant effect influencing 
Tab. 2 Electronic and nuclear relaxation (hyper)polarizabilities of the investigated molecules computed at the CCSD(T)/aug-cc-pVTZ level of theory. All values are given in au.

\begin{tabular}{|c|c|c|c|c|c|c|}
\hline$\omega$ & $\alpha^{\mathrm{EL}}$ & $\alpha^{\mathrm{NR}}$ & $\beta^{\mathrm{EL}}$ & $\beta^{\mathrm{NR}}$ & $\gamma^{\mathrm{EL}}$ & $\gamma^{\mathrm{NR}}$ \\
\hline \multicolumn{7}{|c|}{$\mathrm{CO}_{2}$} \\
\hline 0.00 & 27.05 & 3.09 & - & - & 977 & 1149 \\
\hline 0.08 & 26.44 & 2.99 & - & - & 742 & 948 \\
\hline 0.16 & 25.09 & 2.78 & - & - & 409 & 826 \\
\hline 0.24 & 23.55 & 2.58 & - & - & 210 & 691 \\
\hline \multicolumn{7}{|c|}{$\mathrm{HCCH}$} \\
\hline 0.00 & 30.78 & 0.19 & - & - & 3360 & 1800 \\
\hline 0.08 & 29.55 & 0.17 & - & - & 2588 & 1639 \\
\hline 0.16 & 27.13 & 0.13 & - & - & 1674 & 1206 \\
\hline 0.24 & 24.64 & 0.09 & - & - & 1130 & 926 \\
\hline \multicolumn{7}{|c|}{$\mathrm{HCN}$} \\
\hline 0.00 & 22.53 & 0.14 & 6.23 & -10.42 & 2317 & 911 \\
\hline 0.08 & 21.82 & 0.14 & 6.87 & -10.41 & 1936 & 818 \\
\hline 0.16 & 20.31 & 0.11 & 7.39 & -9.06 & 1359 & 665 \\
\hline 0.24 & 18.66 & 0.08 & 6.70 & -7.67 & 949 & 535 \\
\hline
\end{tabular}

the molecular electrical properties. Recently, a very effective quantum chemical approach, being an extension of the polarizable continuum model for investigations of the effects of extreme pressure on the structural and vibrational properties of molecules, was developed by Cammi et al. ${ }^{49-51}$ The results obtained within this approximation (abbreviated PCMXP) clearly show that the bond distances are shortened and the corresponding harmonic vibrational frequencies are increasing linearly with the magnitude of applied pressure. Similar effects are observed for rigid molecules upon spatial confinement exerted by model potentials. ${ }^{8,15,16,52-56}$ In this context our results presented in the Tab. 1 support the findings reported by other authors, since for all the investigated molecules the same trends are observed.

Short inspection of Tab. 2 shows that both the electronic $\left(P^{\mathrm{EL}}\right)$ and vibrational $\left(P^{\mathrm{NR}}\right)$ contributions to the longitudinal polarizabilities and second hyperpolarizabilities decrease significantly with an increasing strength of spatial confinement $(\omega)$. The plots of relative changes of $\alpha_{z z}$ and $\gamma_{z z z z}$ shown in Fig. 1 indicate that these properties diminish almost linearly with $\omega$, even though initially the rate of this process is somewhat slower due to the character of the applied confining potential. It is interesting to note that the electronic components decrease much faster than the corresponding nuclear relaxation contributions. This indicates that in general the relative importance of the latter should be larger for confined species (cf. Fig. 1). The changes of $P^{\mathrm{EL}}$ and $P^{\mathrm{NR}}$ are usually monotonic, however, there is one exception concerning $\beta^{\mathrm{EL}}$ of HCN molecule. In this case the electronic contribution is initially

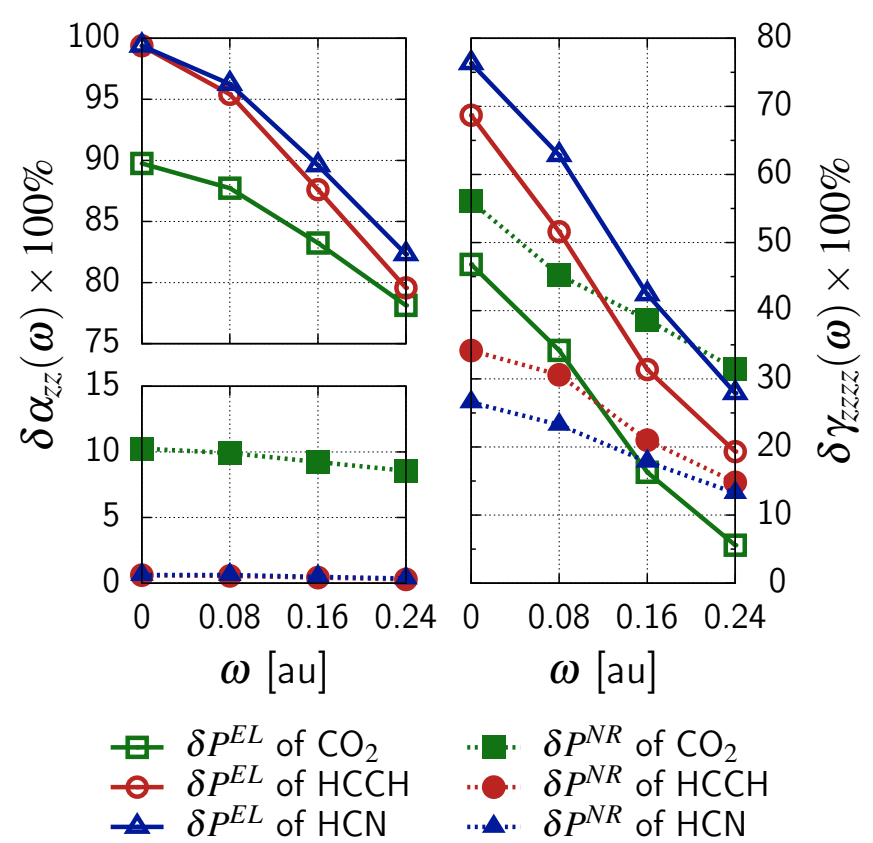

Fig. 1 Relative changes of electronic $\left(P^{\mathrm{EL}}\right)$ and vibrational $\left(P^{\mathrm{NR}}\right)$ contributions to longitudinal polarizabilities (left panel) and second hyperpolarizabilities (right panel) upon applied confining potential. $\delta P^{\mathrm{X}}(\omega)=P^{\mathrm{X}}(\omega) /\left(P^{\mathrm{EL}}(0)+P^{\mathrm{NR}}(0)\right)$.

rising and only under strong confinement starts to diminish. The obtained results are in line with our recent observations related to behavior of the pure electronic first hyperpolarizabilities $\left(\beta^{\mathrm{EL}}\right)$ of HCCCN and OCS molecules for which the values of $\beta^{\mathrm{EL}}$ change non-monotonically as a function of confinement strength. ${ }^{15,19,57}$ However, for the other molecules studied so far ( $\mathrm{LiH}, \mathrm{LiF}, \mathrm{HCl}, \mathrm{HF}$, and $\mathrm{HArF}) \beta^{\mathrm{EL}}$ exhibits different behaviour and decreases upon confinement. ${ }^{13,14,16,18}$

In order to gain an insight into origins of changes of vibrational hyperpolarizabilities upon confinement, the breakdown of $\beta^{\mathrm{NR}}$ and $\gamma^{\mathrm{NR}}$ into harmonic and anharmonic contributions was performed. Harmonic contributions to first (i.e. $[\mu \alpha]^{(0,0)}$ term) and second hyperpolarizability (i.e. $\left[\alpha^{2}\right]^{(0,0)}$ and $[\mu \beta]^{(0,0)}$ terms) were computed based on the BK-PT approach. In that event we used $3 N-5$ normal modes separated from translations and rotations. In the case of $\mathrm{HCN}$ molecule the $[\mu \alpha]^{(0,0)}$ term constitutes $102 \%(\omega=0.0 \mathrm{au})$ and $98 \%(\omega=0.16 \mathrm{au})$ of diagonal nuclear relaxation first hyperpolarizability, thus indicating a negligible net anharmonic contribution $\left(\left[\mu^{3}\right]^{(1,0)}+\left[\mu^{3}\right]^{(0,1)}\right)$. Upon the increase of confinement the harmonic contribution to vibrational first hyperpolarizability is reduced by $17 \%$ and this parallels the drop of $\beta^{\mathrm{NR}}$ by $13 \%$. Much more interesting results were obtained for vibrational contributions to second hyperpolarizability (cf. Fig. 2). Only for carbon dioxide the $[\mu \beta]^{(0,0)}$ 


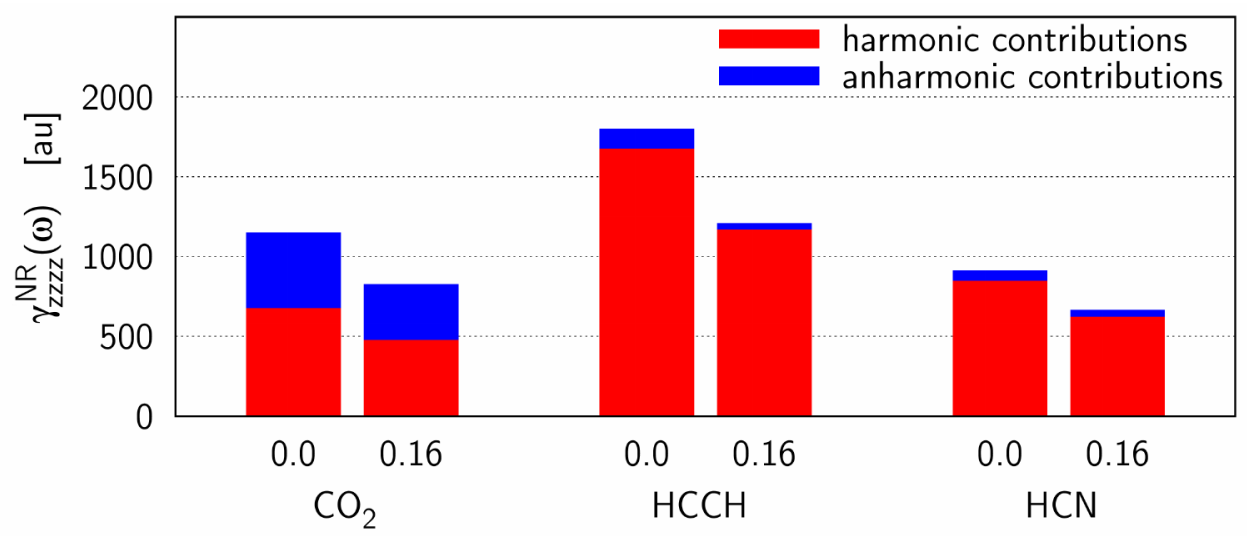

Fig. 2 Breakdown of the longitudinal nuclear relaxation second hyperpolarizability into harmonic and anharmonic contributions upon applied confining potential $(\omega)$.

contribution is significant, i.e. it constitutes $30 \%$ and $40 \%$ of $\left[\alpha^{2}\right]^{(0,0)}$ value for $\omega=0.0$ au and $\omega=0.16 \mathrm{au}$, respectively. For the two other studied molecules, the former is an order of magnitude smaller than the latter. It follows from Fig. 2 that for $\mathrm{HCCH}$ and $\mathrm{HCN}$ the net anharmonic contributions are negligible in comparison with the harmonic ones. The significant change of $\gamma^{\mathrm{NR}}$ upon confinement for these two molecules can thus be predicted by considering only the sum of harmonic contributions. In the case of $\mathrm{CO}_{2}$ molecule the double harmonic model is much less reliable. Our results indicate also that the reduction induced by the confinement is larger for the harmonic terms than for the anharmonic terms.

The most important finding emerging from the analysis of calculated (hyper)polarizabilities (Tab. 2) is that for all investigated molecules the values of total (hyper)polarizabilites $\left(P^{\mathrm{EL}}+P^{\mathrm{NR}}\right)$ are significantly smaller in the presence of spatial restriction than in the case of free molecules. Analysis of the results in terms of signs and absolute magnitudes of the electronic and vibrational contributions to the first and second hyperpolarizabilities leads to the conclusion that both contributions should be considered in the interpretation of the nonlinear optical properties of molecules. This conclusion is particularly important for confined systems since spatial restriction reduces the electronic components to a larger extent than the vibrational contributions. Summing up, the effect of the confinement on the electrical hyperpolarizabilites is larger for the electronic contribution than for vibrational contributions, and larger for harmonic vibrational terms than for anharmonic vibrational terms. Therefore, the importance of vibrational hyperpolarizability increases also with the degree of anharmonicity of vibrational motion.
Acknowledgments: This work was supported by the Project: "Mobilities - enhancing research, science and education at the Matej Bel University", ITMS code: 26110230082, under the Operational Program Education financed by the European Social Fund. W.B. acknowledges financial support from the National Science Centre (Grant No. DEC2013/10/A/ST4/00114). J.M.L. thanks the Spanish Ministerio de Economía y competividad (MINECO, CTQ201452525-P) and the the Generalitat de Catalunya (project number 2014SGR931). This work was also supported by computational grant from ACK Cyfronet. The allocation of computing time is greatly appreciated.

\section{References}

1 R. C. Ashoori, Nature, 1996, 379, 413-419.

2 Advances in Quantum Chemistry: Theory of Confined Quantum Systems, ed. J. R. Sabin, E. J. Brändas and S. A. Cruz, Academic Press, 2009, vol. 57-58, pp. 1-309.

3 J.-P. Connerade and P. Kengkan, Proc. Idea-Finding Symposium, Frankfurt Institute for Advanced Studies, Frankfurt, Germany, 2003, pp. 35-46.

4 J. P. Connerade, Journal of Physics: Conference Series, 2013, p. 012001.

5 M. van Faassen, J. Chem. Phys., 2009, 131, 104108.

6 W. Jaskólski, Phys. Rep., 1996, 271, 1-66.

7 A. L. Buchachenko, J. Phys. Chem. B, 2001, 105, 5839-5846.

8 J. M. Lo, M. Klobukowski and G. H. Diercksen, Advances in Quantum Chemistry, Academic Press, 2005, vol. 48, pp. 59-89.

9 W. Grochala, R. Hoffmann, J. Feng and N. W. Ashcroft, Angew. Chem., Int. Ed., 2007, 46, 3620-3642.

10 V. Schettino and R. Bini, Chem. Soc. Rev., 2007, 36, 869-880.

11 D. G. Billing and A. Katrusiak, Acta Cryst. B Struct. Sci., 2014, 70, 399400.

12 D. S. Chemla, Physics Today, 1993, 46, 46-52.

13 W. Bartkowiak and K. Strasburger, J. Mol. Struct.: THEOCHEM, 2010, 960, 93-97.

14 R. W. Góra, R. Zaleśny, J. Kozlowska, P. Naciążek, A. Roztoczyńska, K. Strasburger and W. Bartkowiak, J. Chem. Phys., 2012, 137, 094307.

15 R. Zaleśny, R. W. Góra, J. Kozłowska, J. M. Luis, H. Ågren and W. Bartkowiak, J. Chem. Theory Comput., 2013, 9, 3463-3472. 
16 J. Kozłowska and W. Bartkowiak, Chem. Phys., 2014, 441, 83-92.

17 A. Roztoczyńska, J. Kozłowska, P. Lipkowski and W. Bartkowiak, Chem. Phys. Lett., 2014, 608, 264-268.

18 J. Kozłowska, R. Zaleśny and W. Bartkowiak, Chem. Phys., 2014, 428, 19-28.

19 A. Kaczmarek, R. Zaleśny and W. Bartkowiak, Chem. Phys. Lett., 2007, 449, 314-318.

20 A. Kaczmarek and W. Bartkowiak, Phys. Chem. Chem. Phys., 2009, 11, 2885-2892.

21 P. K. K. Pandey and D. P. Santry, J. Chem. Phys., 1980, 73, 2899-2901.

22 E. A. Perpete, B. Champagne, J. M. Andre and B. Kirtman, J. Mol. Struct (THEOCHEM), 1998, 425, 115-122.

23 M. Medved, J. Noga, D. Jacquemin and E. A. Perpete, Int. J. Quant. Chem., 2005, 102, 209-223.

24 D. M. Bishop, B. Kirtman, H. A. Kurtz and J. E. Rice, J. Chem. Phys., 1993, 98, 8024-8030.

25 D. M. Bishop and B. Kirtman, J. Chem. Phys., 1991, 95, 2646-2658.

26 D. M. Bishop and E. K. Dalskov, J. Chem. Phys., 1996, 104, 1004-1011.

27 J. L. Andres, J. Bertran, M. Duran and J. Marti, J. Phys. Chem., 1994, 98, 2803-2808.

28 B. Kirtman and B. Champagne, Int. Rev. Phys. Chem., 1997, 16, 389-420.

29 D. M. Bishop, Advances in Chemical Physics, John Wiley \& Sons, Inc., 1998, pp. 1-40.

30 L.-Y. Tang, Z.-C. Yan, T.-Y. Shi and J. F. Babb, Phys. Rev. A, 2014, 90, 012524 .

31 M. Cafiero, L. Adamowicz, M. Duran and J. M. Luis, J. Mol. Struct (THEOCHEM), 2003, 633, 113-122.

32 D. M. Bishop and B. Kirtman, J. Chem. Phys., 1992, 97, 5255-5256.

33 B. Kirtman, J. M. Luis and D. M. Bishop, J. Chem. Phys., 1998, 108, 10008-10012.

34 D. M. Bishop, M. Hasan and B. Kirtman, J. Chem. Phys., 1995, 103, 4157-4159.

35 J. M. Luis, M. Duran, J. L. Andrés, B. Champagne and B. Kirtman, J. Chem. Phys., 1999, 111, 875-884.

36 B. Kirtman, B. Champagne and J. M. Luis, J. Comput. Chem., 2000, 21, 1572-1588.

37 D. Bishop, Adv. Chem. Phys., 1998, 104, 1-40.

38 H. A. Kurtz, J. J. P. Stewart and K. M. Dieter, J. Comput. Chem., 1990, 11, 82-87.

39 M. Medved', M. Stachová, D. Jacquemin, J.-M. André and E. A. Perpète, J. Mol. Struct.: THEOCHEM, 2007, 847, 39-46.

40 M. J. Frisch, G. W. Trucks, H. B. Schlegel, G. E. Scuseria, M. A. Robb, J. R. Cheeseman, G. Scalmani, V. Barone, B. Mennucci, G. A. Petersson, H. Nakatsuji, M. Caricato, X. Li, H. P. Hratchian, A. F. Izmaylov, J. Bloino, G. Zheng, J. L. Sonnenberg, M. Hada, M. Ehara, K. Toyota, R. Fukuda, J. Hasegawa, M. Ishida, T. Nakajima, Y. Honda, O. Kitao, H. Nakai, T. Vreven, J. Montgomery, J. E. Peralta, F. Ogliaro, M. Bearpark, J. J. Heyd, E. Brothers, K. N. Kudin, V. N. Staroverov, R. Kobayashi, J. Normand, K. Raghavachari, A. Rendell, J. C. Burant, S. S. Iyengar, J. Tomasi, M. Cossi, N. Rega, J. M. Millam, M. Klene, J. E. Knox, J. B. Cross, V. Bakken, C. Adamo, J. Jaramillo, R. Gomperts, R. E. Stratmann, O. Yazyev, A. J. Austin, R. Cammi, C. Pomelli, J. W. Ochterski, R. L. Martin, K. Morokuma, V. G. Zakrzewski, G. A. Voth, P. Salvador, J. J. Dannenberg, S. Dapprich, A. D. Daniels, O. Farkas, J. B. Foresman, J. V. Ortiz, J. Cioslowski and D. J. Fox, Gaussian 09, revision C.01, 2009, Gaussian Inc. Wallingford CT.

41 R. R. Wiederkehr and H. G. Drickamer, J. Chem. Phys., 1958, 28, 311316.

42 M. R. Zakin and D. R. Herschbach, J. Chem. Phys., 1986, 85, 2376-2383. 43 M. R. Zakin and D. R. Herschbach, J. Chem. Phys., 1988, 89, 2380-2387. 44 D. Ben-Amotz, M. R. Zakin, H. E. King and D. R. Herschbach, J. Phys. Chem., 1988, 92, 1392-1394.
45 Y. Song, Z. Liu, H.-k. Mao, R. J. Hemley and D. R. Herschbach, J. Chem. Phys., 2005, 122, 174511.

46 M. Jana and S. Bandyopadhyay, Chem. Phys. Lett., 2011, 509, 181-185.

47 L. Pison, M. F. Costa Gomes, A. A. H. Pa?dua, D. Andrault, S. Norman, C. Hardacre and M. C. C. Ribeiro, J. Chem. Phys., 2013, 139, 054510.

48 D. Kajiya and K.-i. Saitow, J. Chem. Phys., 2013, 139, 054509.

49 R. Cammi, V. Verdolino, B. Mennucci and J. Tomasi, Chem. Phys., 2008, 344, 135-141.

50 R. Cammi, C. Cappelli, B. Mennucci and J. Tomasi, J. Chem. Phys., 2012, 137, 154112.

51 M. Pagliai, G. Cardini and R. Cammi, J. Phys. Chem. A, 2014, 118, 5098 5111.

52 R. LeSar and D. R. Herschbach, J. Phys. Chem., 1981, 85, 2798-2804.

53 D. Bielińska-Wąż, G. H. Diercksen and M. Klobukowski, Chem. Phys. Lett., 2001, 349, 215-219.

54 O. Shameema, C. N. Ramachandran and N. Sathyamurthy, J. Phys. Chem. A, 2006, 110, 2-4.

55 A. Sarsa, J. M. Alcaraz-Pelegrina, C. Le Sech and S. A. Cruz, J. Phys. Chem. B, 2013, 117, 7270-7276.

56 R. Colín-Rodríguez and S. A. Cruz, J. Phys. B: At. Mol. Opt. Phys., 2010, 43, 235102.

57 J. Kozłowska, A. Roztoczyńska and W. Bartkowiak, Chem. Phys., 2015, DOI: 10.1016/j.chemphys.2014.12.003. 